\title{
Free totally (anti)symmetric massless fermionic fields in d-dimensional anti-de Sitter space
}

\author{
R. R. Metsaev \\ Department of Theoretical Physics, P. N. Lebedev Physical Institute, Leninsky \\ prospect 53, 117924, Moscow, Russia
}

\begin{abstract}
Free massless fermionic fields of arbitrary spins $s>0$ corresponding to totally (anti)symmetric tensor-spinor representations of the $S O(d-1)$ compact subgroup and in $d$ dimensional anti-de Sitter space are investigated. We propose the free equations of motion, subsidiary conditions and corresponding gauge transformations for such fields. The equations obtained are used to derive the lowest energy values for the above-mentioned representations. A new representation for equations of motion and gauge transformations in terms of generators of anti-de Sitter group $S O(d-1,2)$ is found. It is demonstrated that in contrast to the symmetric case the gauge parameter of the antisymmetric massless field is also a massless field.
\end{abstract}


The long term motivation of our investigation of higher-spin massless field theory in the anti-de Sitter space of higher dimensions is to study the equations of interacting gauge fields of all spins suggested in [1]. Because these equations are formulated in terms of wavefunctions which depend on usual spacetime coordinates and certain twistor variables it is not clear immediately what kind of fields they describe. At present because of [1], it is known that in four dimensions $(d=4)$ they describe unitary dynamics of massless fields of all spins in anti-de Sitter space. It is expected that for the case of higher dimensions $d>4$ they also describe unitary dynamics. The case of half-integral spins, presented here is a necessary step in our study of massless higher spins of all spins for arbitrary $d$. For $d=4$ the integral spins have been studied in [2] and for arbitrary $d$ in [3]. We also hope that our results may be of wider interest, especially to anti-de Sitter supergravity theories.

First let us formulate the main problem that we will solve in this letter. The positive-energy lowest weight irreducible representation of $s o(d-1,2)$ algebra denoted as $D\left(E_{0}, \mathbf{m}\right)$, is defined by $E_{0}$, the lowest eigenvalue of the energy operator, and by $\mathbf{m}=\left(m_{1}, \ldots m_{\nu}\right), \nu=\left[\frac{d-1}{2}\right]$, which is the weight of the $s o(d-1)$ representation. For the case of the four dimensional anti-de Sitter space $(d=4)$ it has been discovered 国 that for fermionic massless fields $E_{0}=s+3 / 2, \mathbf{m}=\left(m_{1}=s+1 / 2\right)$ (see also [5], [6]). In the present work we generalize this result for the case of arbitrary $d$ and for the fermionic fields corresponding to the following two representations of the $s o(d-1)$ algebra. The first representation has $\mathbf{m}_{\text {sym }}=(s+1 / 2,1 / 2, \ldots, 1 / 2)$, which is a totally symmetric representation and the second has $\mathbf{m}_{a s}=(3 / 2, \ldots, 3 / 2,1 / 2, \ldots, 1 / 2)$ (where the $3 / 2$ occurs $s$ times in this sequence) which is a totally antisymmetric one. For gauge fields labeled by $\mathbf{m}_{s y m}$ and $\mathbf{m}_{a s}$ the integer $s$ satisfies $s>0$ and $1<s \leq \nu$, respectively. In this paper we restrict ourselves to the case of even $d$.

Now let us describe our conventions and notation. We describe the anti-de Sitter space as a hyperboloid $\eta_{A B} y^{A} y^{B}=1$ in a $d+1$ - dimensional pseudo-Euclidean space with metric tensor $\eta_{A B}=(+,-, \ldots,-,+), A, B=0,1, \ldots, d-1, d+1$. To simplify our expressions we will drop the metric tensor $\eta_{A B}$ in scalar products.

The generators of the $S O(d-1,2)$ group $J^{A B}$ satisfy the commutation relations

$$
\left[J^{A B}, J^{C D}\right]=\mathrm{i} \eta^{B C} J^{A D}+\text { three terms } .
$$

We split $J^{A B}$ into an orbital part $L^{A B}$ and a spin part $M^{A B}: J^{A B}=L^{A B}+M^{A B}$. The realization of $L^{A B}$ in terms of a differential operator defined on the hyperboloid $y^{A} y^{A}=1$ is

$$
L^{A B}=y^{A} p^{B}-y^{B} p^{A}, \quad p^{A} \equiv \mathrm{i} \theta^{A B} \frac{\partial}{\partial y^{B}}, \quad \theta^{A B} \equiv \eta^{A B}-y^{A} y^{B},
$$

where $p^{A}$ is a momentum operator. The $p^{A}$ has the properties $y^{A} p^{A}=0, p^{A} y^{A}=\mathrm{i} d$ and satisfies the commutation relations $\left[p^{A}, y^{B}\right]=\mathrm{i} \theta^{A B}$ and $\left[p^{A}, p^{B}\right]=\mathrm{i} L^{A B}$. A form for $M^{A B}$ depends on the realization of the representations. We will use the tensor realization of representations. As the carriers for $D\left(E_{0}, \mathbf{m}\right)$ we use totally (anti)symmetric tensorspinor fields $\Psi_{a s(s y m)}^{A_{1} \ldots A_{s}}$ over the hyperboloid $y^{A} y^{A}=1$. Note we do not show explicitly spinor index of $\Psi$. Sometimes it will be convenient to use the generating function 


$$
\left|\Psi_{a s(s y m)}\right\rangle \equiv(s !)^{-1} a_{a s(s y m)}^{A_{1}} \ldots a_{a s(s y m)}^{A_{s}} \Psi_{a s(s y m)}^{A_{1} \ldots A_{s}}|0\rangle,
$$

where $|0\rangle$ is the Fock vacuum (i.e. $\bar{a}^{A}|0\rangle=0$ ) and $a^{A}$ and $\bar{a}^{A}$ satisfy the (anti)commutation relations

$$
\left\{\bar{a}_{a s}^{A}, a_{a s}^{B}\right\}=-\eta^{A B}, \quad\left[\bar{a}_{\text {sym }}^{A}, a_{\text {sym }}^{B}\right]=-\eta^{A B},
$$

and $\left\{a_{a s}^{A}, a_{a s}^{B}\right\}=0,\left[a_{\text {sym }}^{A}, a_{\text {sym }}^{B}\right]=0$. The subscripts as and sym will often be dropped in the following when there is no ambiguity. For realizations given by (1), $M^{A B}$ has the form

$$
M^{A B}=M_{b}^{A B}+\sigma^{A B}
$$

where

$$
M_{b}^{A B}=-\mathrm{i}\left(a^{A} \bar{a}^{B}-a^{B} \bar{a}^{A}\right), \quad \sigma^{A B}=\frac{\mathrm{i}}{4}\left(\gamma^{A} \gamma^{B}-\gamma^{B} \gamma^{A}\right)
$$

and $\gamma^{A}$ are gamma matrices: $\left\{\gamma^{A}, \gamma^{B}\right\}=2 \eta^{A B}$.

Being the carriers for $D\left(E_{0}, \mathbf{m}\right)$, the fields $\Psi$ satisfy the equations

$$
(Q-\langle Q\rangle)|\Psi\rangle=0,
$$

and allow the following subsidiary covariant constraints:

$$
\begin{array}{ll}
y^{A_{1}} \Psi^{A_{1} \ldots A_{s}}=0 & \text { (transversality) } \\
\gamma^{A_{1}} \Psi^{A_{1} \ldots A_{s}}=0 & \\
p^{A_{1}} \Psi^{A_{1} \ldots A_{s}}=0 & \text { (divergencelessness) } \\
\Psi_{\text {sym }}^{A A A_{3} \ldots A_{s}}=0 & \text { (tracelessness) } .
\end{array}
$$

In equation (2) $Q$ is the second-order Casimir operator of the $s o(d-1,2)$ algebra

$$
Q=\frac{1}{2} J^{A B} J^{A B}
$$

while $\langle Q\rangle$ are its eigenvalues for totally (anti)symmetric representations

$$
\begin{aligned}
& \left\langle Q_{a s}\right\rangle=E_{0}\left(E_{0}+1-d\right)+s(d-s)+\frac{1}{8}(d-1)(d-2), \\
& \left\langle Q_{s y m}\right\rangle=E_{0}\left(E_{0}+1-d\right)+s(s+d-2)+\frac{1}{8}(d-1)(d-2) .
\end{aligned}
$$

The $\langle Q\rangle$ can be calculated according to the well known procedure [7]. $E_{0}$ is the lowest eigenvalue of $J^{0 d+1}$. The expression for $Q$ can be rewritten as

$$
Q=p^{2}+L^{A B} M^{A B}+\frac{1}{2} M^{A B} M^{A B}, \quad p^{2} \equiv p^{A} p^{A}
$$

Now taking into account the easily derived equalities $\sigma^{A B} \sigma^{A B}=d(d+1) / 4$ and 


$$
\begin{gathered}
L^{A B} M_{b}^{A B}|\Psi\rangle=-2 s|\Psi\rangle, \quad L^{A B} \sigma^{A B}|\Psi\rangle=\mathrm{i} \not \not p p|\Psi\rangle, \quad \sigma^{A B} M_{b}^{A B}|\Psi\rangle=s|\Psi\rangle \\
M_{b}^{A B} M_{b}^{A B}\left|\Psi_{(a s) \text { sym }}\right\rangle=\left\{\begin{array}{l}
2 s(d+1-s)\left|\Psi_{a s}\right\rangle \\
2 s(s+d-1)\left|\Psi_{\text {sym }}\right\rangle
\end{array}\right.
\end{gathered}
$$

$\not p^{2}=p^{2}+\mathrm{i} \not \not \not p$, where $\not p \equiv \gamma^{A} p^{A}$ and $\not y \equiv \gamma^{A} y^{A},\{\not p, \not y\}=\mathrm{i} d$, one can rewrite equation (2) as

$$
\left(\not p^{2}-m^{2}\right)|\Psi\rangle=0
$$

where we introduce formally the "mass" term

$$
m^{2} \equiv\left(E_{0}+\frac{1}{2}\right)\left(E_{0}+\frac{1}{2}-d\right)
$$

To define $E_{0}$ corresponding to massless fields we should respect gauge-invariant equations of motion for $\Psi$. For the case of totally symmetric representations we could use the interesting results of [8] (see also [9]), where the gauge-invariant equations were formulated in terms of linear curvatures. However, we prefer to use a simpler and as it seems to us more adequate procedure to derive $E_{0}$. We proceed in the following way.

First we write the most general gauge transformations for $\Psi^{A_{1} \ldots A_{s}}$

$$
\delta \Psi^{A_{1} \ldots A_{s}}=\sigma(A)\left(p^{A_{1}} \Lambda^{A_{2} \ldots A_{s}}+y^{A_{1}} R^{A_{2} \ldots A_{s}}+\gamma^{A_{1}} S^{A_{2} \ldots A_{s}}\right)+\text { cyclic permutations }
$$

where $\Lambda^{A_{2} \ldots A_{s}}, R^{A_{2} \ldots A_{s}}$, and $S^{A_{2} \ldots A_{s}}$ are the totally (anti)symmetric tensor-spinor fields. In equation (9) and below 'cyclic permutations' indicates the terms which are obtainable by making cyclic permutations of indices $A_{1}, \ldots, A_{s}$. For totally symmetric representations $\sigma_{\text {sym }}(A)=1$ while for the antisymmetric one we have $\sigma_{a s}(A)=1$ for even cyclic permutations of $\left(A_{1} \ldots A_{s}\right)$ and $\sigma_{a s}(A)=(-1)^{s-1}$ for odd cyclic permutations of $\left(A_{1} \ldots A_{s}\right)$. On the gauge parameter fields $\Lambda^{\cdots}, R^{\cdots}$ and $S^{\cdots}$ we impose the algebraic constraints like (3)-(6):

$$
\begin{aligned}
& y^{A_{2}} \Lambda^{A_{2} \ldots A_{s}}=y^{A_{2}} R^{A_{2} \ldots A_{s}}=y^{A_{2}} S^{A_{2} \ldots A_{s}}=0, \\
& \gamma^{A_{2}} \Lambda^{A_{2} \ldots A_{s}}=\gamma^{A_{2}} R^{A_{2} \ldots A_{s}}=\gamma^{A_{2}} S^{A_{2} \ldots A_{s}}=0, \\
& p^{A_{2}} \Lambda^{A_{2} \ldots A_{s}}=p^{A_{2}} R^{A_{2} \ldots A_{s}}=p^{A_{2}} S^{A_{2} \ldots A_{s}}=0 \\
& \Lambda_{\text {sym }}^{A A A_{4} \ldots A_{s}}=R_{\text {sym }}^{A A A_{4} \ldots A_{s}}=S_{\text {sym }}^{A A A_{4} \ldots A_{s}}=0 .
\end{aligned}
$$

Secondly, we require that subsidiary conditions (3)-(6) and (7) be invariant with respect to the gauge transformation (9). Note that due to (10)-(13) the invariance requirement of (6) is already satisfied. From the invariance requirement of (3) (i.e. $\left.y^{A_{1}} \delta \Psi^{A_{1} \ldots}=0\right)$ it follows

$$
R^{A_{2} \ldots A_{s}}=\mp \mathrm{i}(s-1) \Lambda^{A_{2} \ldots A_{s}}-y S^{A_{2} \ldots A_{s}} .
$$


In equation (14) and in the following upper and lower signs correspond to antisymmetric and symmetric cases, respectively. Thus at this stage we have the gauge transformations

$$
\delta \Psi^{A_{1} \ldots A_{s}}=\sigma(A)\left(\left(p^{A_{1}} \mp \mathrm{i}(s-1) y^{A_{1}}\right) \Lambda^{A_{2} \ldots A_{s}}+\theta^{A_{1} B} \gamma^{B} S^{A_{2} \ldots A_{s}}\right)+\text { cyclic permutations }
$$

From the invariance requirement of (四) with respect to (15) (i.e. $\gamma^{A_{1}} \delta \Psi^{A_{1} \cdots}=0$ ) we get

$$
(\not p \mp \mathrm{i}(s-1) y) \Lambda^{A_{2} \ldots A_{s}}+(d \mp(2 s-2)) S^{A_{2} \ldots A_{s}}=0 .
$$

From the invariance requirement of (司) (i.e. $p^{A_{1}} \delta \Psi^{A_{1} \ldots}=0$ ) we get

$$
\left(p^{2}-(s-1)(s-1 \mp(d-1))\right) \Lambda^{A_{2} \ldots A_{s}}+(\not p \pm \mathrm{i}(s-1 \mp d) y) S^{A_{2} \ldots A_{s}}=0 .
$$

From the invariance requirement of (可) (i.e. $\left(\not p^{2}-m^{2}\right) \delta \Psi^{\cdots}=0$ ) we get

$$
m_{\text {as }}^{2}=s(s-d), \quad m_{\text {sym }}^{2}=(s-2)(s+d-2) .
$$

In deriving (18) we use the commutation relations

$$
\begin{aligned}
& {\left[\not p^{2}, p^{A}\right]=(1-d) p^{A}-2 \mathrm{i} y^{A} p^{2}+\gamma^{A} \not p,} \\
& {\left[\not p^{2}, y^{A}\right]=2 \mathrm{i} p^{A}+(d-1) y^{A}+\gamma^{A} \not y,} \\
& {\left[\not p^{2}, \theta^{A B} \gamma^{B}\right]=y^{A}(-2 \mathrm{i} \not p-(d+1) \not y)+\gamma^{A} .}
\end{aligned}
$$

Now comparing (18) and (8) it is seen that there is a quadratic equation for $E_{0}$, whose solutions read

$$
E_{0}^{(1,2)}=\left\{\begin{array}{llll}
d-s-1 / 2 & \text { or } & s-1 / 2 & \text { for } \Psi_{a s} \\
s+d-5 / 2 & \text { or } & -s+3 / 2 & \text { for } \Psi_{s y m}
\end{array}\right.
$$

As seen from (19) there exists an arbitrariness of choosing $E_{0}$. Thus we can conclude that the gauge invariance by itself does not uniquely determine the physical relevant value of $E_{0}$ (in this regard the situation is similar to the $d=4$ case, see [2]). To choose a physical relevant value of $E_{0}$ one can exploit the unitarity condition, that is, (i) Hermiticity $\left(J^{A B}\right)^{\dagger}=J^{A B}$; (ii) the positive norm requirement. Making use of this unitarity condition one can prove that the $E_{0}$ should satisfy the inequalities $E_{0} \geq d-s-1 / 2$ for the case of $\Psi_{a s}$ and $E_{0} \geq s+d-5 / 2$ for the case of $\Psi_{\text {sym }}$. Comparing these inequalities with (19) we find the following physical relevant values of $E_{0}$

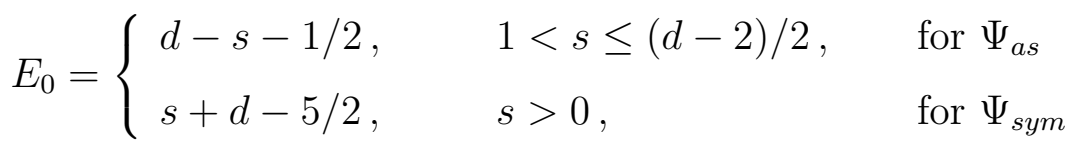

where we recall domains of values of $s$. Thus the analysis anti-de Sitter gauge transformations in combination with unitarity enables us to fix the $E_{0}$, i.e. gauge invariance and unitarity complement each other. For the case of $d=4$ and $\Psi_{\text {sym }}$ the solution 
$E_{0}=s+3 / 2$ is the well known result of 4 . Note that our result for $E_{0}(20)$ cannot be extended to cover the case of one-half spinor representation $(s=0)$ because the $E_{0}$ obtained are relevant only for gauge fields. This case should be considered in its own right and the relevant value $E_{0}$ (for $\left.s=0\right)=(d-1) / 2$ can be obtained by using requirement of conformal invariance (see [11]). The case of $s=0, d=4$ has been investigated in [12].

Up to now we analysed second-order equations for $\Psi$. Now we would like to derive first-order equations. To do that we use the relations

$$
\begin{gathered}
\not p=-\mathrm{i} \not \boldsymbol{k}, \quad[\kappa, \not y]=\not y(-2 \kappa-d), \quad y^{2}=1, \\
\not p^{2}=\kappa^{2}+d \kappa, \quad \kappa \equiv \sigma^{A B} L^{A B}
\end{gathered}
$$

and rewrite equation (7) as follows:

$$
\left(\kappa+E_{0}+\frac{1}{2}\right)\left(\kappa-E_{0}-\frac{1}{2}+d\right)|\Psi\rangle=0
$$

Thus we could use the following two first-order equations of motion:

$$
\begin{gathered}
\left(\kappa-E_{0}-\frac{1}{2}+d\right)|\Psi\rangle=0, \\
\left(\kappa+E_{0}+\frac{1}{2}\right)|\tilde{\Psi}\rangle=0 .
\end{gathered}
$$

Due to relation

$$
\left(\kappa-E_{0}-\frac{1}{2}+d\right)|\Psi\rangle=-\not y\left(\kappa+E_{0}+\frac{1}{2}\right) y|\Psi\rangle
$$

it is clear that $|\Psi\rangle$ and $|\tilde{\Psi}\rangle$ are related by

$$
|\Psi\rangle=y|\tilde{\Psi}\rangle
$$

i.e. equations (22) and (23) are equivalent. We will use the equation of motion given by (22). Now we should verify gauge invariance of the first-order equation (22) with respect to gauge transformations (15). It turns out that the invariance requirement of (22) with respect to (15) leads to

$$
S^{A_{2} \ldots A_{s}}=0
$$

In deriving (24) we use the commutation relations

$$
\begin{gathered}
{\left[\kappa, p^{A}\right]=\gamma^{A} \not p-p^{A}, \quad\left[\kappa, y^{A}\right]=\gamma^{A} \not y-y^{A}} \\
{\left[\kappa, \theta^{A B} \gamma^{B}\right]=2 \mathrm{i} p^{A} y+(d-1) y^{A} \not y+\gamma^{A} .}
\end{gathered}
$$

Thus the final form of gauge transformations is

$$
\delta \Psi^{A_{1} \ldots A_{s}}=\sigma(A)\left(\left(p^{A_{1}} \mp \mathrm{i}(s-1) y^{A_{1}}\right) \Lambda^{A_{2} \ldots A_{s}}\right)+\text { cyclic permutations }
$$


which can be rewritten in a simpler form

$$
\delta|\Psi\rangle=a^{A}\left(p^{A} \mp \mathrm{i}(s-1) y^{A}\right)|\Lambda\rangle
$$

or in terms of $E_{0}$ (see equation (20)) as follows:

$$
\delta|\Psi\rangle=a^{A}\left(p^{A}+\mathrm{i}\left(E_{0}+\frac{3}{2}-d\right) y^{A}\right)|\Lambda\rangle,
$$

where we use the notation

$$
|\Lambda\rangle=((s-1) !)^{-1} a^{A_{2}} \ldots a^{A_{s}} \Lambda^{A_{2} \ldots A_{s}}|0\rangle .
$$

The equations of motion for $\Lambda$ can be obtained from (16) and (24)

$$
(\kappa \pm(s-1))|\Lambda\rangle=0
$$

which can also be rewritten in terms of $E_{0}$

$$
\left(\kappa-E_{0}-\frac{3}{2}+d\right)|\Lambda\rangle=0 .
$$

Making use of (24) and (27) one can make sure that equation (17) is also satisfied.

Thus we have constructed equations of motion (22) which respect gauge transformations (26), where the gauge parameter fields $\Lambda$ satisfy the constraints (10)-(13) and equations of motion (28). The relevant values of $E_{0}$ are given by (20). Note that the equations of motion are written in terms of the operator $\kappa$ (see equation (21)). The $\kappa$ was introduced in [10 while constructing the equation of motion for the field associated with the representation labeled by $D\left(E_{0}, 1 / 2\right)$. The $\kappa$ is expressible in terms of the orbital part $L^{A B}$ (see equation (21)). Now we would like to rewrite our equations of motion in terms of complete angular momentum $J^{A B}$. In our opinion such a form is more promising. To do that let us first multiply equations (22) and (28) by $y$. Then we use the following equalities:

$$
\begin{gathered}
y^{A} a^{B} J^{A B}=(a p) \mp \mathrm{i}(y a)(a \bar{a})+\mathrm{i} a^{2}(y \bar{a})-\frac{\mathrm{i}}{2} a^{A} \theta^{A B} \gamma^{B} y, \\
y^{A} \gamma^{B} J^{A B}=-\mathrm{i} \not y\left(\kappa+\frac{1}{2} d\right)+y^{A} \gamma^{B} M_{b}^{A B},
\end{gathered}
$$

where the expressions like $(a b)$ indicate the scalar product $a^{A} b^{A}$. Now making use of the equalities $(a \bar{a})|\Psi\rangle=-s|\Psi\rangle,(a \bar{a})|\Lambda\rangle=-(s-1)|\Lambda\rangle$ which tell us that $\Psi$ and $\Lambda$ are tensors of rank $s$ and $s-1$, respectively, and the equalities $(y \bar{a})|\Psi\rangle=0,(y \bar{a})|\Lambda\rangle=0$ which are the constraints (3) and (10) we rewrite the equations of motion (22) as

$$
\left(y^{A} \gamma^{B} J^{A B}+\mathrm{i}\left(E_{0}-\frac{d-1}{2}\right) y\right)|\Psi\rangle=0,
$$

the gauge transformations (26) as

$$
\delta|\Psi\rangle=y^{A}\left(a^{B}-\frac{a^{C} \theta^{C D} \gamma^{D}}{2 E_{0}+3-d} \gamma^{B}\right) J^{A B}|\Lambda\rangle,
$$


and equations of motion for gauge parameter fields (28) as

$$
\left(y^{A} \gamma^{B} J^{A B}+\mathrm{i}\left(E_{0}^{\Lambda}-\frac{d-1}{2}\right) y\right)|\Lambda\rangle=0
$$

where we introduce lowest energy value for $\Lambda$ : $E_{0}^{\Lambda}=E_{0}+1$

$$
E_{0}^{\Lambda}= \begin{cases}d-s+1 / 2 & \text { for } \Lambda_{a s} \\ s+d-3 / 2 & \text { for } \Lambda_{s y m}\end{cases}
$$

In deriving (30) we use the equations of motion for $\Lambda$ (28). Note also that in deriving of (29)-(31) it is necessary to use the relevant values of $E_{0}$ given by (20).

With the values for $E_{0}^{\Lambda}$ at hand we are ready to provide an answer to the question: do the gauge parameter fields meet the masslessness criteria? As expected, by analogy with $d=4$ case the answer for the case of $\Lambda_{\text {sym }}$ is negative. As for $\Lambda_{a s}$ the answer is positive. Indeed the inter-relations between of spin value $s$ and energy value $E_{0}$ for massless fields are given by (20). Therefore the energy values for spin $s-1$ massless fields are obtainable by making substitutions $s \rightarrow s-1$ in (20). Because of the relations

$$
\begin{array}{ll}
E_{0}^{\Lambda}=E_{0}(s \rightarrow s-1) & \text { for } \Lambda_{a s} \\
E_{0}^{\Lambda} \neq E_{0}(s \rightarrow s-1) & \text { for } \Lambda_{s y m}
\end{array}
$$

we conclude that the $\Lambda_{a s}$ is a massless field while the $\Lambda_{\text {sym }}$ is a massive field. The result that $\Lambda_{\text {sym }}$ is a massive field is a generalization of well known results for $d=4$. To our knowledge the masslessness of $\Lambda_{a s}$ has not been referred to previously in the literature. Note that this fact was not discovered in $d=4$ because for this case there are no essentially totally antisymmetric representations, i.e. for the totally symmetric case one exhausts all representations.

In conclusion, let us formulate the results of this letter. For massless fermionic fields of arbitrary spins $s>0$ corresponding to totally (anti)symmetric tensor-spinor representations of the $S O(d-1)$ compact subgroup and in $d$-dimensional anti-de Sitter space we have constructed: (i) free equations of motion (22), subsidiary conditions (3)(6), corresponding gauge transformations (26), and constraints (10)-(13) and equations of motion for the gauge parameter fields (28); (ii) the energy lowest values (20); (iii) the new representation for equations of motion (29), (31) and gauge transformations (30) in terms of the generators of the anti-de Sitter group $S O(d-1,2)$. It is demonstrated that in contrast to the symmetric case the gauge parameter of the antisymmetric massless field is also a massless field (see equations (33) and (34)).

This work was supported in part by INTAS, Grant No.94-2317, by the Russian Foundation for Basic Research, Grant No.96-01-01144, and by the NATO Linkage, Grant No.931717. 


\section{References}

[1] Vasiliev M A 1990 Phys. Lett.B 243 378; 1991 Phys. Lett.B 257 111; 1991 Class. Quantum Grav. 81387

[2] Fronsdal C 1975 Phys.Rev.D 12 3819; 1979 Phys.Rev.D 20848

[3] Metsaev R R 1994 Class.Quanum Grav. 11 (1994) L141; 1995 Phys.Lett.B 35478

[4] Fronsdal C and Haugen R B 1975 Phys.Rev.D 123810

[5] Fang J and Fronsdal C 1980 Phys.Rev.D 221361

[6] Breitenlohner P and Freedman D Z 1982 Ann.Phys. 144249

[7] Perelomov A M and Popov V S 1968 Sov.J.Nucl.Phys. 7460

Barut A O and Raczka R, Theory of Group Representations and Applications (PWN-Polish Scientific Publishers, Warszawa 1977)

[8] Vasiliev M A 1988 Nucl.Phys.B 30126

[9] Lopatin V E and Vasiliev M A 1988 Mod.Phys.Lett.A 3257

[10] Dirac P A M 1935 Ann.Math. 36657

[11] Metsaev R R 1995 Mod.Phys.Lett.A 101719

[12] Deser S, Kay J and Stelle K 1977 Phys.Rev.D 162445 